BULLETIN (New Series) OF THE AMERICAN MATHEMATICAL SOCIETY

Volume 35, Number 2, April 1998, Pages 145-156

S 0273-0979(98)00744-7

\title{
THE UNIVERSAL TEMPLATES OF GHRIST
}

\author{
R. F. WILLIAMS
}

\begin{abstract}
This is a report on recent work of Robert Ghrist in which he shows that universal templates exist. Put another way, there are many structurally stable flows in the 3 -sphere, each of which has periodic orbits representing every knot type. This answers a question raised originally by Mo Hirsch and popularized by the contrary conjecture by Joan Birman and the present author.
\end{abstract}

\section{INTRODUCTION}

It is a pleasant task to report on the recent thesis of Rob Ghrist. Contrary to our conjectures, he has shown that some structurally stable flows in $S^{3}$ contain all knot types as periodic orbits. This includes many of the flows studied by us and others; with the possible exception of M. Sullivan, these people had no inkling of this fact. His basic trick is to prove much more - that these flows contain all closed braids as unions of periodic orbits. There are at least two more "tricks" in this excellent work: a systematic search for "sub-templates" and nice use of symbolic dynamics to understand what Ghrist calls, "deeply lying orbits". In effect, one must sidestep many orbits corresponding to "short words" in the four symbols used to gain a structure in which the property of containing all braids is transparent.

This report is structured as follows: we begin with enough basic definitions to enable us to state the problem carefully and give a taste of the recent history, mostly personal. Then we proceed straight to the constructions.

\section{DeFinitions}

There is a large literature in dynamics which has some pertinence to the work of Ghrist: structurally stable flows, hyperbolic structure, etcetera. We can, however, in the interest of space, get by here with little foundational material, since the problem solved by Ghrist was already well formulated. This by no means reduces the interest in his work.

Templates (also called knot holders) were introduced in [B-W1] and have been used to characterize, geometrically, the periodic orbits of structurally stable flows in 3-dimensions as well as to to study the Lorenz attractor [W4]. This disparity accounts for some variation in the terminology; here we follow the usage of Ghrist. Though symbolic dynamics is crucial in much of the earlier work, the relation

Received by the editors June 3, 1997, and in revised form August 10, 1997, and January 21, 1998.

1991 Mathematics Subject Classification. Primary 57-XX.

Supported in part by a grant from the National Science Foundation.

Thanks to Robert Ghrist for his help, in particular for help in drawing the figures.

We thank the Mathematics Department of Montana State University for their hospitality.

(C)1998 American Mathematical Society 
between a template, a sub-template and their two sets of symbolic dynamics is especially important here; in ferreting out this relationship, he takes a slightly different point of view.

A template is a branched 2-manifold [W1] with boundary, lying in $S^{3}$, which is endowed with a smooth vector field. At certain portions of the boundary (called "gaps" below), the vector field is outwardly transverse, so that the orbits of this field leave the branched surface. Thus there is a smooth semi-flow defined on the invariant set of all points which never exits the template; this set is one dimensional. In more detail, a template is the union of strips, and each strip is a copy of the standard flow box in dimension 2:

$$
\left\{(x, y) \in \mathbb{R}^{2}: 0 \leq x, y \leq 1\right\}
$$

endowed with the constant vector field $\frac{\partial}{\partial x}$ at each point. Where the strips meet, along branches, the vectors coincide so that a unique semi-flow is determined. The copying homeomorphism stretches the $x=1$ end so that the resulting flow, where defined, is expanding. Two or more of these strips are assembled into a template, so that the resulting flow is well defined in the positive direction, but at the branches is not well defined for negative time.

As we shall see in figure 2, the template consists of two strips, $x$ and $y$, so that the flow on the left side passes around to the left and back down to the branch. Similarly, the flow on the right passes around to the right behind the strip $x$ and back down to the branch. There is a middle portion at each branch, called a gap, at which the orbits leave the template. Orbits which leave are no longer of interest to us. Note that the periodic orbits never leave. The dark horizontal line is a branch; above it two planar pieces come together where they are tangent to each other. Thus each point on a branch lies in two smooth disks which coincide below the branch, but are disjoint above. Each branch in a template is homeomorphic to the unit interval $[0,1]$ and called a branch line. Each branch line is the union of closed line segments separated by gaps, which in turn are open line intervals. The collection of all branch segments in a template $T$ is denoted by $\beta(T)$. Thus the semi-flow on a template is transverse to the branches and has no rest points.

Two templates (or links) are separable provided there is an isotopy of $S^{3}$ which takes one of them inside the unit 2-sphere and the other outside. A collection of templates (or links) is said to be completely separable provided there is a collection of disjoint 3-disks and an isotopy of $S^{3}$ which takes each of the templates into one of the 3-disks, and no two of them into the same 3-disk. Finally, a knot $K$ is said to factor into knots $K_{1}, K_{2}$ both knotted provided there is a tame 2-sphere $S^{2}$ such that $S^{2} \cap K$ consists of two points $A, B$, such that $\alpha \cup\left(\left(\right.\right.$ interior $\left.\left.S^{2}\right) \cap K\right)=K_{1}$ and such that $\alpha \cup\left(\left(\right.\right.$ exterior $\left.\left.S^{2}\right) \cap K\right)=K_{2}$, for any arc $\alpha$ on $S^{2}$ joining $A$ and $B$. The unknot plays the role of 1 and is not allowed as a prime. Schubert proved the unique factorization of knots into primes in the 1950s.

\section{SOME PERTINENT History, MOSTLy PERSONAL}

As a lifelong enthusiast of knots, the present author was finally allowed to think about them when, studying the periodic orbits of the Lorenz attractor (for another purpose [W4]), it was noticed that they were mostly knotted, [W3]. This was an exciting period: we got to play with these knots, with string, with pencil and paper, and a contraption that a colleague dubbed a "knot loom"; we found a lot of torus knots and met lots of knot theorists (one — Siebenmann — wondered if the Lorenz 
template contained all knots). DeWitt Sumners was helpful early on, and told me about algebraic (in the sense of singularities) knots. John Conway was also helpful though doubtful that the knot loom could be marketed. But it was Joan Birman who knew about positive braids (those in which the generators $\sigma_{i}$ occur with only positive exponents; see below), and braids in general, that put us in business; we wrote two papers together, in the second of which we related a question, originated by that great interrupter of talks, Mo Hirsch:

Conjecture 1. [B-W2] There is no template that contains all knot types as periodic orbits.

The second conjecture was a specific recipe for proving the first one:

Conjecture 2. Each template $T$ has a bound $N_{T}$, such that no knot on $T$ could have more than $N_{T}$ prime factors.

This was in part based on the false security we found working on the Lorenz template, for all periodic orbits found there are [W5] prime knots.

This conjecture was exploded in M. Sullivan's thesis, [S1].

Theorem. [S1] The template $V$ (see below) contains composite knots with arbitrarily many factors.

Sullivan's technique was to show that $V$ contains disjoint copies $L^{\prime}$ of the Lorenz template, and $V^{\prime}$ of $V$ itself. It follows that any knot in $V^{\prime}$ can be joined to any knot in $L$, and thus, by induction, that $V$ contains composite knots with arbitrarily many factors. Ghrist's technique is a far-reaching development of this idea.

In the second paper [B-W2], we made a study of the flow induced by the fibration of the complement of the figure 8 knot, $K$. That is, the fibration has the circle as base, and thus choosing a metric, one can lift the obvious "constant" flow on the circle to $S^{3}-K$, and can even extend this flow to have $K$ as a periodic orbit.

Conjecture 3. Except for the knot $K$ itself, this flow has no other figure 8 knot as periodic orbit.

This conjecture was based on circumstantial evidence: though the 5-knot, close cousin to the figure 8 , is easy to find, lots of searching yielded no figure $8 \mathrm{~s}$.

Braids, a brief discussion. A braid consists of a certain number $s$ of strands, drawn in the plane, but pictured in 3 dimensions, beginning at a top level and descending monotonically to a bottom level, crossing one another in a certain pattern. (See figure 1, in which $s=3$.) The strands are numbered left to right. Braids on $s$ strands form a group $B_{s}$ under concatenation, with generators $\sigma_{i}, i=1, \ldots s-1$. Here $\sigma_{i}$ is the braid in which the only crossing is of the $i$ th strand over the $i+1$ st strand and $\sigma_{i}^{-1}$ is similar, except that the $i$ th strand crosses under the $i+1$ st strand. Thus left handed crossings are chosen to be positive, though many authors make the other choice. A braid $\beta$ is made into a link, called the closure of $\beta$, by joining the bottom of the strands to the top with no further crossing. In figure 1 we have indicated the braid $\sigma_{1} \sigma_{2}^{-1} \sigma_{1} \sigma_{2}^{-1}$ and its closure which is the figure 8 knot. Alexander proved that every link $L$ can be represented as the closure of a braid; hence the minimum possible number of strands in such a representation is an invariant, called the braid index of $L$. 


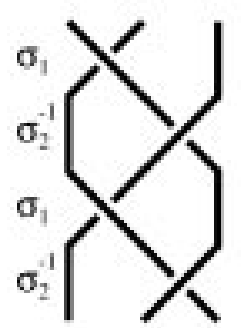

BRAID

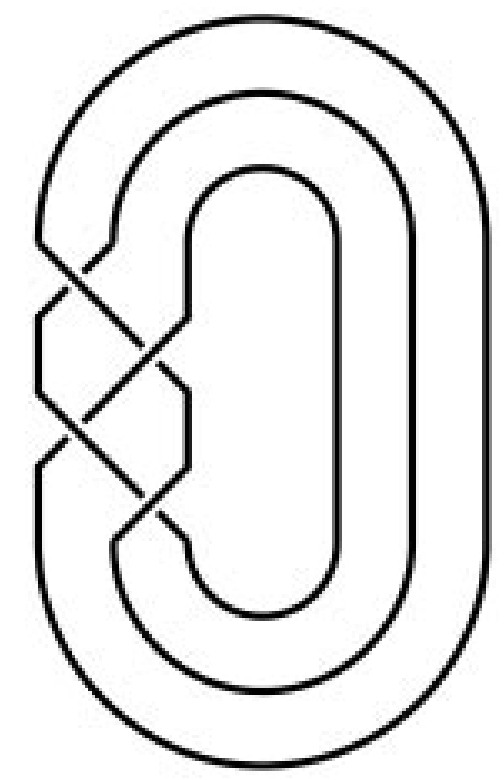

FiguRe 1

The excitement gained momentum at the advent of the "Jones' polynomial", (a.k.a. the "HOMFLY" polynomial) which has proved so important, especially in the case of braids. Until Jones' work, the braid index — so natural to the dynamicists, as flows are essentially already braided — was almost useless, as so little was known about it.

\section{The templates $U, V$, and $W_{q}$}

The simplest template is perhaps the Lorenz template, figure 2, with two strips, $x, y$. Templates $U$ and $V$ (figure 3 ) are just a bit more complicated, the crucial difference being that their orbits contain both left and right crossings. Each has four strips, labeled $1,2,3,4$, or $x_{1}, x_{2}, x_{3}, x_{4}$. In the end, we learn that $U$ and $V$ are essentially equivalent to each other, but Ghrist utilizes their apparent difference (by embedding each into the other) to prove his result.

Any point $P$ on a branch is uniquely determined by the infinite orbit under the semi-flow, beginning at $P$. This orbit passes successively through, say, strips

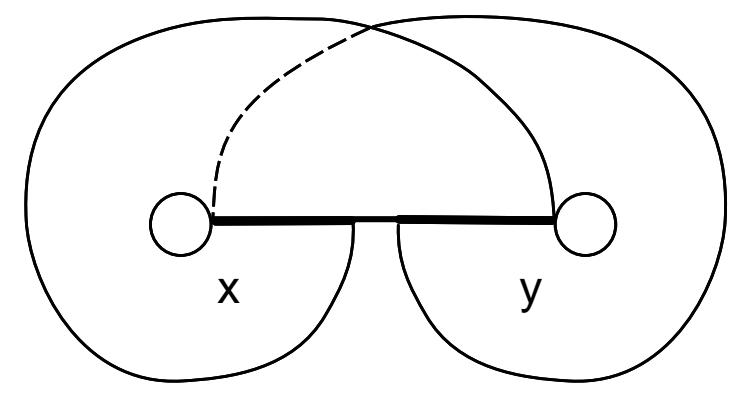

FiguRe 2 

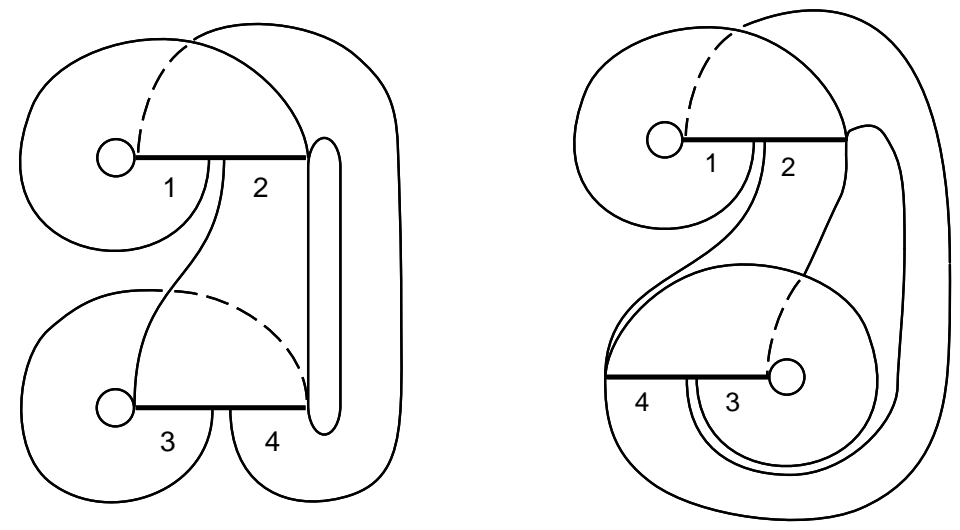

Figure 3. Templates V (left) and U

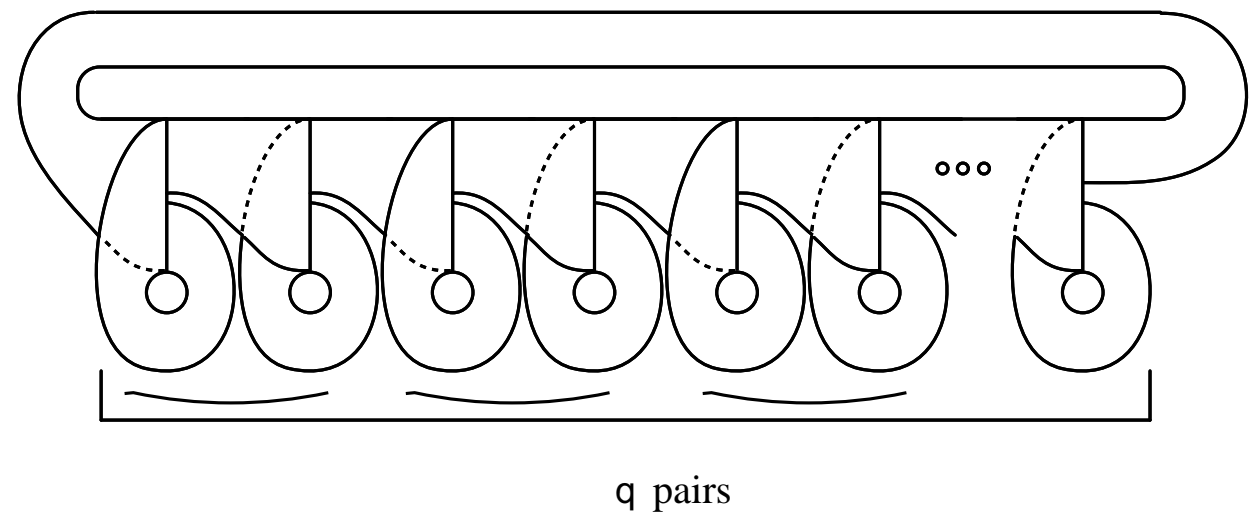

FiguRE 4

$x_{1}, x_{2}, \ldots$ so that this point is labeled by the symbol $x_{1} x_{2} x_{3} \ldots$. Then each orbit in either $U$ or $V$ that remains for all time without exiting corresponds to an infinite sequence of $x_{i}$ s, for example, $x_{1}^{\infty}$ or $1^{\infty}$. Similarly, $13(42)^{\infty}$. This notation is crucial below, where geometric embeddings are difficult to describe with pictures. Thus, for example, the four segments of either $U$ or $V$ can be described by their boundaries, with notation $\partial_{i}^{l}(V)$ for left boundary of the $i$ th segment of $V$, and so on. For example, $\partial_{4}^{l}(V)=(42)^{\infty}$. That is, the periodic orbit passing successively through strip 4 , then strip 2 , then 4 , etc.

The template $W_{q}$, figure 4 , has $q$ pairs of "ears", where each pair consists of, first, one with positive crossings and next one with negative crossings; thus $W_{q}$ has $4 q$ strips and is a $q$-fold covering of $V$. Note first that any consecutive sequence of four ears on $W_{q}$ contains the elements $\pi_{i}=\sigma_{1} \ldots \sigma_{i}$, and $\pi_{i}^{\prime}=\sigma_{1}^{-1} \ldots \sigma_{i}^{-1}, i=1, \ldots n$ of the braid group, $B_{n+1}$. (See figure 5 , in which $\pi_{2}, \pi_{2}^{\prime}$ in $B_{5}$ are illustrated.) These elements, $\pi_{i}, \pi^{\prime}$ are easily seen to generate $B_{n+1}$.

Thus if $\beta$ is a braid which can be expressed in $B_{n+1}$ with $m$ of these generators, then $\beta$ lies in $W_{q}$, for $q \geq 4 m$. Thus any braid on $N$ strands lies in $W_{q}$, for $q$ sufficiently large. 


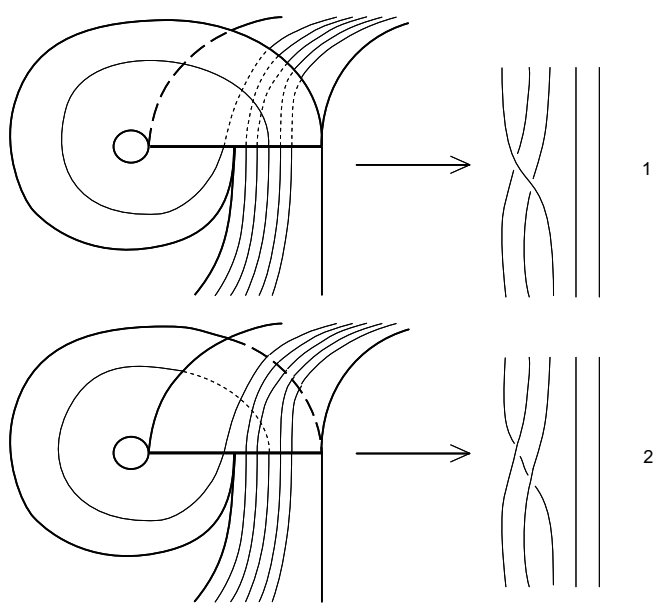

FiguRE 5

This just about exhausts the easy, general abstract nonsense of the proof. Our goal is to show

Theorem. $W_{q} \subset V$, for all $q$.

This will complete the proof that $V$ is a universal template, and is done in the remaining sections.

\section{Template inflations}

There are several ways of putting one template into another. First, a template inflation of a template $S$ into a template $T$ is a map $R: S \rightarrow T$, taking orbits to orbits, which is a diffeomorphism onto its image. One notes that the image of such an inflation is a sub-template of $T$, and says that $R$ is isotopic if this image is embedded in $S^{3}$ just as $S$ is.

Using this language, two rather elementary isotopic inflations, $F: U \rightarrow V$ and $G: V \rightarrow U$ (see figures 6 and 7) are the key "legs" that carry Ghrist. (Sullivan knew of $G$ but not $F$ and thus could only hop, to paraphrase Douady.) Note that in Ghrist's rendition, each of the branch symbols of the domain is "inflated" into a "word" in the range symbols.

Thus in symbolic dynamics, the inflation $F$ is given by

$$
x_{1} \mapsto x_{1}, \quad x_{2} \mapsto x_{1} x_{2} x_{3}, \quad x_{3} \mapsto x_{4} x_{2}, \quad x_{4} \mapsto x_{4},
$$

and $G$ is given by

$$
x_{1} \mapsto x_{1}, \quad x_{2} \mapsto x_{2}, \quad x_{3} \mapsto x_{2} x_{4}, \quad x_{4} \mapsto x_{2} x_{3} x_{4} .
$$

Thus we have what symbolic dynamicists call a "block" map between the respective 1-sided shifts. These block maps compose by substitution and, moreover, are essentially equivalent to the inflation itself,

Next, the inflation $\chi$ is introduced, which sends each orbit into its mirror image. The beauty is that composing two reflections yields an isotopy, so that the conjugates - note that $\chi$ is an involution $-F^{*}=\chi F \chi$ and $G^{*}=\chi G \chi$ are isotopic inflations. These are the principal ingredients used to show that there is an 

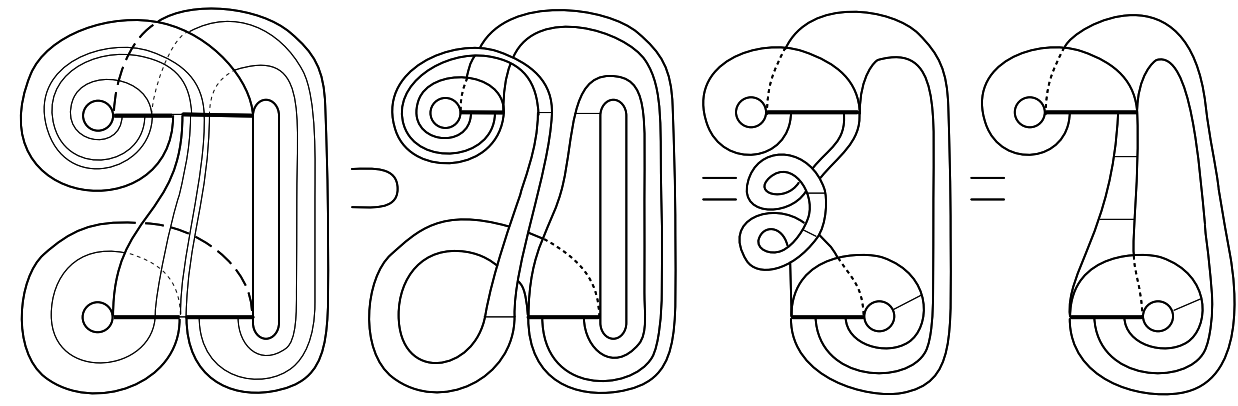

Figure 6 . The inflation $F$ is isotopic
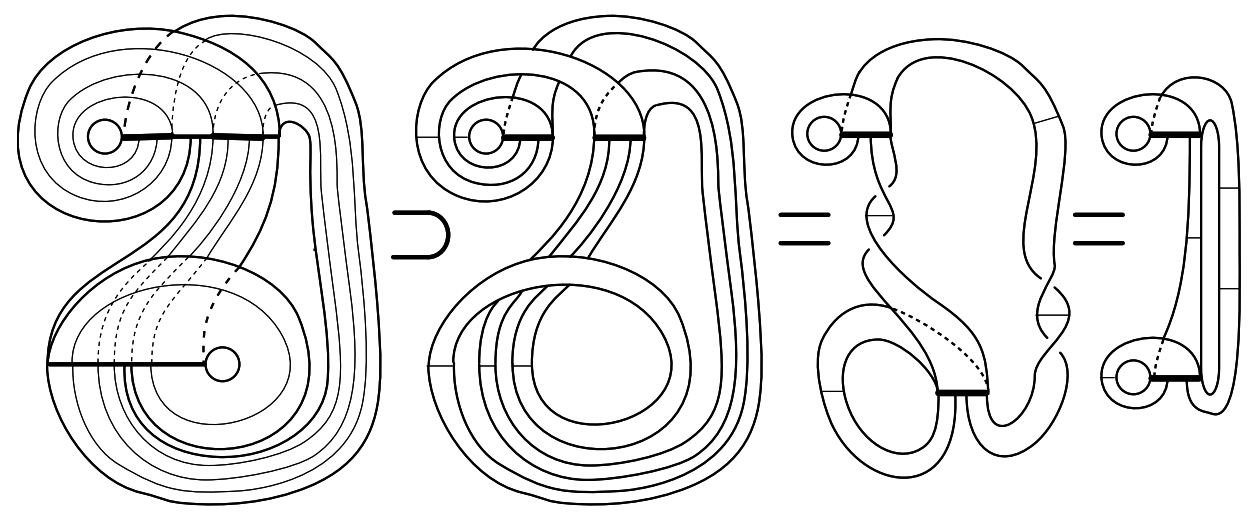

FIgURE 7 . The inflation $G$ is isotopic

isotopic inflation of $W_{q}$ into $V$ for any $q$. The block map for $\chi$ (for either $U$ of $V$ ) is as follows:

$$
x_{1} \mapsto x_{3}, \quad x_{2} \mapsto x_{4}, \quad x_{3} \mapsto x_{1}, \quad x_{4} \mapsto x_{2} .
$$

As a preliminary result,

Lemma. $G(V)$ and $G^{*}(V)$ are disjoint except for the common boundary $\left(x_{1} x_{2} x_{3} x_{4}\right)^{\infty}$ and are separable inflations.

Proof. This can be seen directly from the picture, figure 8 . One easily checks that they are disjoint except for the boundary orbit, $\left(x_{1} x_{2} x_{3} x_{4}\right)^{\infty}$, via symbolic dynamics. That is, the branch sets of $G(V)$ are

$$
\left[1^{\infty}, 1(1234)^{\infty}\right], \quad\left[1(24)^{\infty},(1234)^{\infty}\right], \quad\left[(24)^{\infty}, 24(2341)^{\infty}\right], \quad\left[2341^{\infty},(2341)^{\infty}\right] .
$$

Whereas the branch sets of $G^{*}(V)$ are

$$
\left[(42)^{\infty}, 42(4123)^{\infty}\right], \quad\left[4123^{\infty},(4123)^{\infty}\right], \quad\left[3^{\infty}, 3(3412)^{\infty}\right], \quad\left[3(42)^{\infty},(3412)^{\infty}\right]
$$

The sub-templates to the left are black and grey; to the right we have collapsed the transverse direction to aid in seeing that they are separated. 

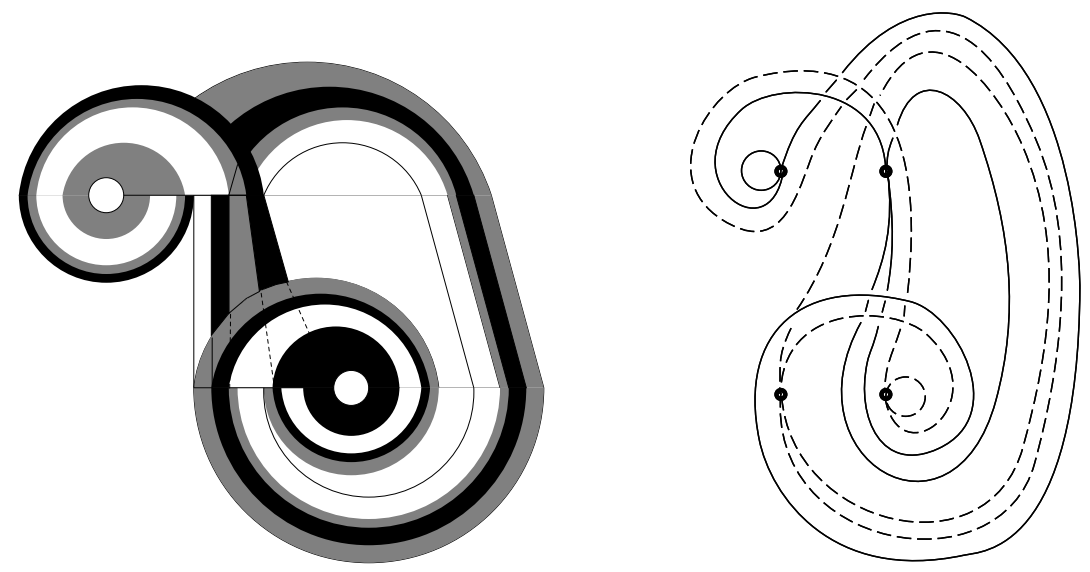

Figure 8

Corollary. Each template $U, V$ contains infinitely many sub-templates isotopic to $U$ and $V$ which are pairwise disjoint and completely separable.

Proof. Define the isotopic inflation $A_{n}$ by $A_{n}=F G\left(F G^{*}\right)^{n}$, for each $n$. Then the image of each $A_{n}$ is disjoint and separable from the image of $A_{n+k}, k>0$, for note that $A_{n+k}$ factors as

$$
A_{n+k}=\left[F G\left(F G^{*}\right)^{k-1}\right]\left(F G^{*}\right)^{n+q 1},
$$

so that the image of $A_{n+k}$ is contained in the image of $\left(F G^{*}\right)^{n+1}$. Then by the proposition, the images of $A_{n}$ and $\left(F G^{*}\right)^{n+1}$ are disjoint and separable, as they differ by changing one $G$ to a $G^{*}$.

\section{The Principal Lemma}

Principal Lemma. Let $S$ be a sub-template of $V$ and let $I$ be the component of $S \cap l^{1}(V)$ which is leftmost among all intersections on the upper branch line. If $\partial^{l}(I) \neq x_{1}^{\infty}$, then $S$ is contained in a sub-template $S^{+} \subset V$; this sub-template is isotopic to $S$ except for the addition of an un-knotted ear along $I$, and $S^{+}$contains the orbit $\partial_{4}^{l}(V)$.

Proof. The sub-template $S$ is completely determined by its branch set, $\beta(S)$. That is, flowing a branch segment forward until it completely covers a set of two or more branch segments sweeps out the corresponding strip. Thus we proceed to construct

$$
\beta\left(S^{+}\right)=\beta(S) \cup\left\{\left[x_{1}^{\infty}, x_{1} \partial^{r}(I)\right], I\right\} .
$$

To make this into a template, whenever some endpoint of some branch line of $\beta\left(S^{+}\right)$ ends in $\partial^{l}(I)$, replace it with $x_{1}^{\infty}$. The resulting template is as in figure 9 . Note in particular that the appended ear is completely separated from the rest of the template, and that in thickening up the incoming strip along $x_{4}$, we include the orbit $\partial_{4}^{l}(V)$ in $S^{+}$.

We next show how negative ears are added.

Lemma. Let $S$ be a sub-template of $V$ and let $I$ be the component of $S \cap l^{2}(V)$ which is minimal among all intersections on the lower branch line. If $\partial^{l}(I) \neq x_{1}^{\infty}$, 

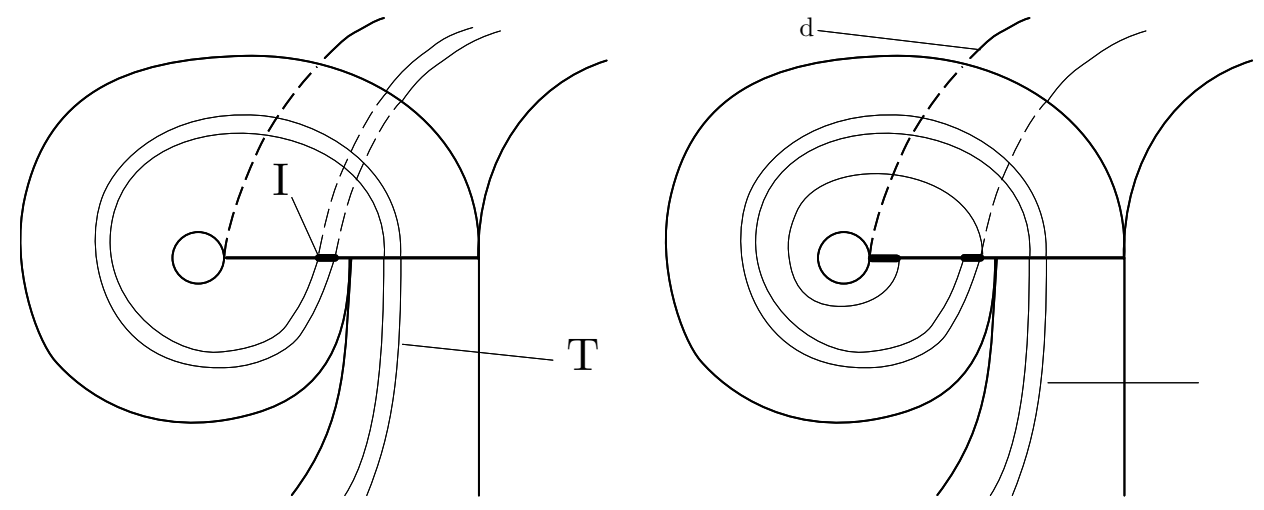

FiguRE 9

then $S$ is contained in a sub-template $S^{-} \subset V$; this template is isotopic to $S$ except for the addition of an un-knotted ear along $I$, and $S^{+}$contains the orbit $\partial_{2}^{l}(V)$.

Proof. This is completely "dual" to the last lemma, and is proved by applying the inflation $\chi$ to $V$, adding a positive ear, then applying $\chi$ again, resulting in the desired negative ear.

Since the inflations $F$ and $G$ tend to put the templates $U$ and $V$ "deep" inside, composing these inflations puts them even deeper. The next result is the core of Ghrist's construction; the difficulty in its proof lies in the fact that the inflations used are too deep to see geometrically.

Proposition. Consider the inflation $H=F^{*} G F G^{*}: V \rightarrow V$. The minimal point of $H(V) \cap l_{1}(V)$ is contained in the orbit $H\left(\partial_{2}^{l}(V)\right)$.

Proof. We resort to symbolic dynamics, and to avoid clutter we only write the subscripts. Then the block map for $H$ is

$$
\begin{aligned}
& 1 \longmapsto 23341(24)^{2} 2341 \\
& 2 \longmapsto 23341(24)^{3} 2341 \\
& 3 \longmapsto 2334124 \\
& 4 \longmapsto 2334124
\end{aligned}
$$

Thus the left boundary points of the four branch lines map as follows

$$
\begin{aligned}
\partial_{1}^{l}(V)=1^{\infty} & \longmapsto\left(23341(24)^{2} 2341\right)^{\infty} \\
\partial_{2}^{l}(V)=1(24)^{\infty} & \longmapsto 23341(24)^{3} 2341(2334124)^{\infty} \\
\partial_{3}^{l}(V)=3^{\infty} & \longmapsto(2334124)^{\infty} \\
\partial_{4}^{l}(V)=41^{\infty} & \longmapsto 2334124\left(23341(24)^{2} 2341\right)^{\infty}
\end{aligned}
$$

and the right boundary points map later in the order than the corresponding left ones. Consider the symbol

$$
\sigma^{14} H\left(\partial_{2}^{l}(V)\right)=1(2334124)^{\infty},
$$


where $\sigma$ is the shift operator. As we are using 1-sided shifts here, $\sigma$ just drops the first symbol. We claim it is minimal in $l_{1}(V)$ among all shifts of the image of every other endpoint of $\beta(V)$ which begin with a 1 . To see this by hand is not a big task, as our symbolic dynamics is, by design, so simple. (One could use a computer.)

Finally note that the appended ear is quite simple and separated from the rest of the template. Also, the orbit $\partial_{4}^{l}(V)$ is included in $S^{+}$, since this is the orbit that arrives at the periodic orbit, $x_{1}^{\infty}$.

We have just managed to append a positive ear, and proceed to append a negative one.

Proposition. Consider the inflation $H^{*}=F G^{*} F^{*} G: V \rightarrow V$. The minimal point of $H^{*}(V) \cap l_{2}(V)$ is contained in the orbit $H^{*}\left(\partial_{4}^{l}(V)\right)$.

Proof. Now we can apply $\chi$ to the minimal word in the last lemma to get

$$
\chi \sigma^{14} H\left(\partial_{2}^{l}(V)\right)=\chi\left(1(2334124)^{\infty}\right)
$$

is minimal in $\chi\left(l_{1}(V)\right.$. As $\chi$ commutes with the shift, we get

$$
\left.\sigma^{14} \chi H\left(\partial_{2}^{l}\right)\right)=3(4112342)^{\infty}
$$

is minimal in $l_{2}(V)$. Inserting $\chi$ conveniently, we get

$$
\sigma^{14} \chi H \chi\left(\chi \partial_{2}^{l}(V)\right)=\sigma^{14} H^{*}\left(\partial_{4}^{l}(V)\right)=3(4112342)^{\infty}
$$

is minimal in $l_{2}(V)$.

Proposition. For each $q$ there is an isotopic inflation of $W_{q} \subset V$, and thus both $U$ and $V$ are universal templates.

Proof. As we will be working with a series of distinct copies of the template $V$, we introduce some notation. Let $\left\{V_{i}\right\}$ denote a sequence of distinct copies of the embedded template $V$ - each is embedded in a different copy of $S^{3}$. Construct a

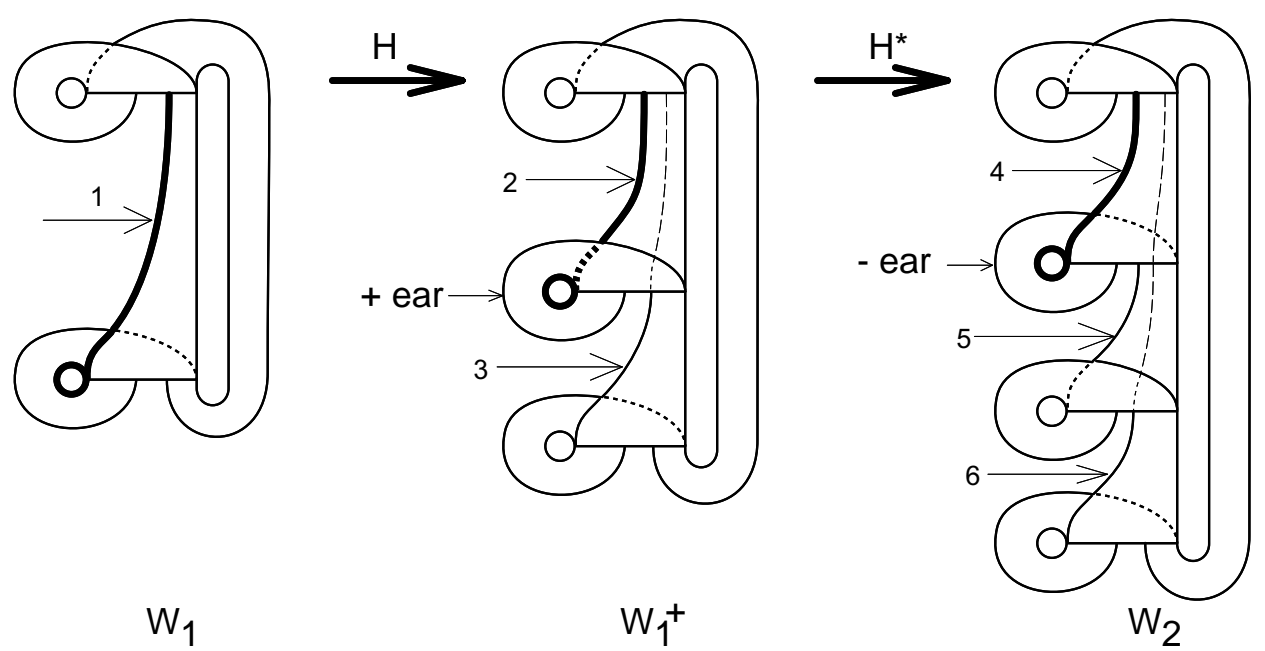

Figure 10. Key: $\mathbf{1}=\partial_{2}^{l}\left(V_{1}\right) . \quad \mathbf{2}=\partial_{2}^{l}\left(V_{1}\right) . \quad \mathbf{3}=H\left(\partial_{2}^{l}\left(V_{1}\right)\right)$. $\mathbf{4}=\partial_{2}^{l}\left(V_{3}\right) . \quad \mathbf{5}=H\left(\partial_{4}^{l}\left(V_{2}\right)\right) . \quad \mathbf{6}=H^{*} H\left(\partial_{2}^{l}\left(V_{1}\right)\right)$. 
sequence of templates and isotopic inflations in which the maps alternate between $H$ and $H^{*}$.

$$
V_{1} \longrightarrow V_{2} \longrightarrow V_{3} \longrightarrow V_{4} \longrightarrow \ldots
$$

Then we may append a positive ear to $H\left(V_{1}\right)$ creating the template $W^{+} \subset V_{2}$. Then by mapping the template $V_{2}$ into $V_{3}$ via $H^{*}$ a negative ear may be appended to $H^{*}\left(W^{+}\right)$along $H^{*}\left(\partial_{4}^{l}\left(V_{2}\right)\right.$. Since the negative ear is appended along an interval having endpoint on $H^{*}\left(\partial_{4}^{l}\left(V_{2}\right)\right.$, this ear precedes the positive ear (in the sense of the flow direction), yielding a sub-template of $V_{3}$ isotopic to $W_{1}$. Continuing this construction, we obtain a sub-template of $V_{2 q+1}$ isotopic to $W_{q}$. See figure 10 .

This completes the proof.

\section{Some UnSOLVED PRoblems}

1. Ghrist has this idea about characterizing universal templates: A template $T$ is universal iff it contains an infinite collection of pairwise separable unknots, each of which is untwisted (i.e., the normal bundle of these is an annulus which bounds a disk in $S^{3}$ ).

2. Find a fluid flow (solution to Navier-Stokes or Euler equations) which has flow lines tracing out all knots.

3 . There are only countably many templates. Yet there are obviously uncountably many families of knots. Which families of knots and links occur as the set of all periodic orbits in some template? The answer should be in terms of knot theory invariants.

4. More specific. Characterize Lorenz knots - knots which appear as periodic orbits on the Lorenz template.

5. Very specific: The finite aperiodic words $w$ in two symbols, $x, y$, correspond 1to- 1 with the periodic orbits $K_{w}$ of the Lorenz attractor. Show that the genus of $K_{x w}$ is not less than the genus of $K_{w}$, provided $w$ and $x w$ are aperiodic

\section{REFERENCES}

[B-W1] Birman, J. and Williams, R.F., Knotted periodic orbits in dynamical systems I: Lorenz's equations, Topology 22 ((1)) (1983), 47-82. MR 84k:58138

[B-W2] _ Knotted periodic orbits in dynamical systems, II: Knot holders for fibered knots, Contemporary Mathematics 20 (1983), Amer. Math. Soc., Providence, RI, pp. 1-60. MR 86a:58084

[F] Franks, J., Knots, links, and symbolic dynamics, Annals of Math. 113 (1981), 529-552. MR 83h:58074

[F-W] Franks, J. and Williams, R.F., Entropy and knots, Transactions American Mathematical Society 291 ((1)) (1985), 241-253. MR 86k:58105

[G] Ghrist, R., Branched two-manifolds supporting all links, Topology 36(2) (1997), 423-448. MR 98b:57009

[G-H] Ghrist, R. and Holmes, P., Knots and orbit genealogies in three dimensional flows, Bifurcations and periodic orbits of vector Fields, NATO ASI series C, vol. 408, Kluwer Academic Press, 1993, pp. 185-239. MR 95g:58192

[G-H-S] R. Ghrist, P. Holmes, and M. Sullivan, Knots and links in three-dimensional flows, Lecture notes in Mathematics, vol. 1654, Springer, 1997. CMP 98:04

[H-W] Holmes, P. and Williams, R.F., Knotted periodic orbits in suspensions of Smale's horseshoe: torus knots and bifurcation sequences, Archive for Rational Mechanics and Analysis 90 ((2)) (1985), 115-193. MR 87h:58142

[S1] Sullivan, M.C., Prime decomposition of knots in Lorenz-like templates, J. Knot Theory and Its Ramifications 2 ((4)) (1993), 453-462. MR 94k:57017 
[S2] _ The prime decomposition of knotted periodic orbits in dynamical systems, J. Knot Theory and Its Ramifications 3 ((1)) (1994), 83-120. MR 95b:57012

[W1] Williams, R. F., Expanding attractors, Colloque de Topologie Differentielle, Mont-Aiguall 1969, Universite de Montpellier, 1969, pp. 79-89. MR 44:4784

[W2] _ Expanding attractors, Publicationes des Institute des Hautes Etudes Scientifique 43 (1974), 169-203. MR 50:1289

[W3] , The structure of Lorenz attractors, Publications Mathematique I.H.E.S. 50 (1979), 321-347. MR 82b:58055b

[W4] Lorenz knots are prime, Ergodic theory and dynamical systems 4 (1983), 147163. MR 86c:58103

[W5] , The structure of Lorenz attractors, Turbulence Seminar, Springer Lecture Notes, vol. 615, 1977, pp. 94-112. MR 57:1566

Department of Mathematics, The University of Texas at Austin, Austin, Texas 787121082

E-mail address: bob@math.utexas.edu

Current address: The Institute for Advanced Study, Princeton, New Jersey 08540

E-mail address: bobwill@math.ias.edu 